\title{
PROCESAMIENTO DE CLÁUSULAS RELATIVAS ANIDADAS, MEMORIA OPERATIVA Y ENVEJECIMIENTO*
}

\author{
NESTED RELATIVES CLAUSES PROCESSING, WORKING \\ MEMORY, AND AGING
}

\author{
BEATRIZ ARANCIBIA GUTIÉRREZ \\ Universidad Católica de la Santísima Concepción. Concepción, Chile \\ barancib@ucsc.cl \\ MÓNICA VÉLIZ \\ Universidad de Concepción. Concepción, Chile \\ mveliz@udec.cl \\ BERNARDO RIFFO \\ Universidad de Concepción. Concepción, Chile \\ bernardo@udec.cl \\ RUBÉN ROA-URETA \\ ruben.roa.ureta@gmail.com
}

\section{RESUMEN}

Este estudio examina los efectos del envejecimiento, la memoria operativa y la complejidad sintáctica en el procesamiento de oraciones. Con este propósito se compara el desempeño de adultos mayores y adultos jóvenes en una tarea de lectura autoadministrada de oraciones con anidamiento de cláusulas de relativo sujeto y objeto. Se efectuó un análisis de efectos mixtos para los tiempos de lectura, el acierto a preguntas y tiempos de respuesta, que toma en cuenta el hecho de tener observaciones repetidas en los individuos y la distribución de probabilidad correcta para las variables observadas. Los resultados muestran efectos de la edad en el procesamiento, pero no respaldan la hipótesis de un declive en el procesamiento sintáctico como efecto de una menor capacidad de memoria operativa en los adultos mayores. Además, el análisis confirma la complejidad de las relativas objeto en comparación con las relativas sujeto en español.

Palabras clave: Envejecimiento, memoria operativa, complejidad sintáctica, procesamiento sintáctico, cláusulas relativas.

\footnotetext{
* Investigación financiada por el proyecto Fondecyt 1050591.
} 


\begin{abstract}
This study examines the effects of age, working memory and syntactic complexity on sentence processing. For this purpose we compare the performance of younger and older adults in a self-administered reading task of sentences with subject and object nested relatives clauses. A mixed effects analysis was carried for reading times, accuracy and response times, which took into account that observations were repeated inside individuals and the correct probability distribution of the observed variables. The results show effects of age in processing, but they do not support the hypothesis of syntactic processing decline as an effect of less working memory capacity in older adults. In addition, the data analysis confirms the complexity of object relatives processing compared to subject relatives processing in Spanish.
\end{abstract}

Keywords: Aging, working memory, syntactic complexity, syntactic processing, relative clauses.

Recibido: 19/06/2013. Aceptado: 14/01/2014.

\title{
I. INTRODUCCIÓN
}

\subsection{Envejecimiento, MO y procesamiento sintáctico}

- xiste abundante literatura que señala que se producen cambios en el desem-

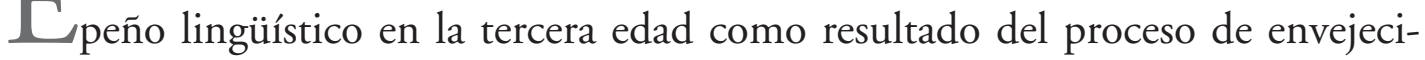
miento cognitivo normal que afecta a las personas mayores (para una revisión, véase Véliz, Riffo y Arancibia, 2010). Dichos cambios se asocian a una declinación gradual de la actuación cognitiva que no afecta a todas las habilidades de la misma manera ni altera la capacidad del individuo para interactuar con el medio (Baltes y Baltes, 1990; Buckner, 2004; Gil, 2000; Burke y Shafto, 2008; Jurado, Matute y Rosselli, 2008).

Se han elaborado diversas explicaciones sobre las causas del declive cognitivo en la vejez normal. La más general y tal vez uno de los fenómenos mejor establecidos en relación con el envejecimiento cognitivo es el enlentecimiento general de la velocidad de transmisión neural (Cerella, 1985; Salthouse, 1985, 1996). Una explicación de carácter más específico alude a la disminución de la capacidad funcional de la memoria operativa (MO). Desde esta perspectiva, muchas de las diferencias que se producen en el desempeño cognitivo de las personas mayores son interpretadas como signos de la pérdida de flexibilidad y eficiencia del componente responsable del control ejecutivo de este sistema de memoria, posiblemente relacionadas con el deterioro de la efectividad de los sistemas frontales del cerebro (Buckner, 2004; Jurado et al., 2008). 
En este estudio nos proponemos examinar si el envejecimiento afecta el procesamiento sintáctico y si, de ser así, esto tiene relación con la reducción de la capacidad de $\mathrm{MO}$ que experimentan los adultos mayores. Aunque este problema ha originado gran cantidad de investigación empírica y un sostenido debate, constituye todavía un campo de investigación abierto, en el cual se entrecruzan cuestiones de orden teórico y metodológico. Una de las más controversiales tiene que ver con la existencia de dos concepciones distintas de la organización de los recursos de $\mathrm{MO}$ implicados en el procesamiento sintáctico: la teoría de recursos compartidos (Just y Carpenter, 1992; King y Just, 1991) y la teoría de recursos de memoria divididos (Caplan y Waters, 1999).

De acuerdo con la teoría de recursos compartidos, la MO cuenta con un conjunto único de recursos de memoria operativa verbal, cuya amplitud es determinante para efectuar de manera eficiente las operaciones que subyacen a la comprensión de oraciones. Los postulados fundamentales de esta visión, fuertemente influenciada por el modelo de Baddeley (1986; Baddeley y Hitch, 1974), son los siguientes: la comprensión lingüística involucra operaciones de almacenamiento y cómputo de información, una fuente única de recursos limitados sirve a ambas funciones y diferencias individuales en la capacidad operativa de este sistema se relacionan con diferencias en la comprensión lingüística (Just y Carpenter, 1992). Basados en esta posición, diversos autores han sostenido la hipótesis de que una disminución de recursos de $\mathrm{MO}$ en la vejez permite predecir un declive en el procesamiento de estructuras sintácticas complejas (Carpenter, Miyake y Just, 1994; Craick, Anderson, Kerr y Li, 1995; Kemper, 1992; Kemper y Kemptes, 1999). Esto se manifestaría en una reducción tanto de la cantidad de información que los sujetos pueden mantener activa en un momento dado del procesamiento como del número de cómputos sintácticos que pueden realizar durante ese mismo periodo (Carpenter et al., 1994). Esto sería más evidente en oraciones cuya sintaxis obliga a mantener activa por más tiempo parte de la información a la espera de completar los cómputos intermedios.

El enfoque teórico descrito ha recibido respaldo empírico de numerosos estudios en los cuales los adultos mayores obtienen tasas más bajas de desempeño que los jóvenes en tareas que requieren comprensión o recuerdo inmediato de oraciones de sintaxis compleja. Entre estos estudios se cuentan los de Cheung y Kemper (1992), Kemper (1987), Kemper, Greiner, Marquis, Prenovost y Mitzner (2001), Kynette y Kemper (1986), Obler, Fein, Nicholas y Albert (1991). En años más recientes, Goral, Clark-Cotton, Spiro, Obler, Verkuilen y Albert (2011) también aportan evidencias del papel predictor de la $\mathrm{MO}$ de los adultos mayores en tareas de juicio metalingüístico sobre oraciones complejas. Por su parte, Kail, Lemaire y Lecacheur (2012) relacionan el declive que experimenta la $\mathrm{MO}$ en la vejez con los resultados obtenidos por sus sujetos en una tarea experimental consistente en leer oraciones y detectar en línea la presencia de violaciones sintácticas. 
Caplan y Waters (1999) sostienen en su teoría de recursos de memoria divididos que la $\mathrm{MO}$ verbal cuenta con recursos especializados para las tareas inconscientes, obligatorias y automáticas que forman parte del procesamiento interpretativo. Dichas tareas corresponden a las conversiones acústico-fonéticas, el reconocimiento de palabras, el acceso léxico y la construcción de las representaciones prosódicas, sintácticas, semánticas y discursivas. Los procesos post-interpretativos, por su parte, dependerían de recursos de propósito general. Estos procesos abarcan las operaciones conscientes y controladas que ocurren fuera de curso, cuando se recupera el significado ya extraído para usarlo en tareas mediadas verbalmente. En el caso de la comprensión de oraciones, se trata de tareas en que los sujetos deben recuperar de su memoria las oraciones, revisar su estructura sintáctica o ciertos aspectos del análisis para responder alguna pregunta o emitir un juicio.

Siguiendo su teoría, Caplan y Waters (1999) defienden la idea de que el procesamiento sintáctico subyacente a la comprensión de oraciones se mantendría a buen resguardo del declive que provoca el envejecimiento cognitivo en el funcionamiento de la MO. Consistentes con este planteamiento son los resultados de estudios que usan metodologías on-line, en los que no se encuentran suficientes evidencias de una relación entre $\mathrm{MO}$ y procesamiento sintáctico inmediato en las personas mayores (Caplan y Waters, 1999; Caplan, Dede, Waters, Michaud y Tripodis, 2011; Clifton, Traxler, Mohamed, Williams, Morris y Rayner, 2003; Dede, Caplan, Kemtes y Waters, 2004; Kemmer, Coulson, De Ochoa y Kutas, 2004; Kemper, Crow y Kemptes, 2004; Kemptes y Kemper, 1997; Waters y Caplan, 1999, 2001). Por lo tanto, un punto interesante en el debate es la tensión que se genera entre las metodologías on-line o en curso y las off-line o fuera de curso. $\mathrm{Al}$ respecto, Waters y Caplan (1999) sostienen que las evidencias de declinación sintáctica en la vejez se han encontrado principalmente a través de métodos offline, lo que reflejaría dificultades para llevar a cabo tareas que requieren procesamiento post-interpretativo, posiblemente como consecuencia de la reducción de los recursos de memoria de propósito general. Ambas capacidades, procesamiento inmediato y procesamiento a posteriori, serían entonces independientes.

Existen también enfoques alternativos para explicar la preservación de las habilidades de procesamiento en la tercera edad. Uno que ha ido cobrando fuerza en los últimos años se basa en la hipótesis de la compensación (Cabeza, Grady, Nyberg, McIntosh, Tulving y Kapur, 1997; Cabeza, 2002; Cabeza, Anderson, Locantore y McIntosh, 2002). Esta sostiene que, gracias a su plasticidad, el cerebro no experimenta de manera pasiva los cambios propios del envejecimiento, sino que responde poniendo en funcionamiento determinados mecanismos compensatorios o de adaptación funcional. Reflejo de esto sería la reducción de la asimetría entre ambos hemisferios y la propagación menos focalizada de la respuesta neuronal (Cabeza, 2002; Escobar, 2001), lo cual contribuiría a preservar las habilidades cognitivas. En el ámbito específico del procesamiento de oraciones, evidencias 
obtenidas con potenciales evocados y técnicas de neuroimagen dan cuenta de estos cambios en el funcionamiento cerebral, los que se han asociado a una mejor actuación de los adultos mayores durante la ejecución de diversas tareas on line y off line de procesamiento sintáctico (Cabeza, 2002; Grossman, Cooke, DeVita, Alsop, Detre, Chen y Gee, 2002; Kemmer et al., 2004; Park y Payer, 2001; Payer, Marshuetz, Sutton, Hebrank, Welsh y Park, 2006; Tyler, Shafto, Randall, Wright, Marslen-Wilson y Stamatakis, 2010).

Un marco explicativo relacionado con la hipótesis de la compensación es el que ofrecen Stine-Morrow y colaboradores (Stine-Morrow, Ryan y Leonard, 2000; Stine-Morrow, Miller y Hertzog, 2006a; Stine-Morrow, Shake, Miles y Noh, 2006b; Stine-Morrow, 2007; Stine-Morrow, Parisi, Morrow, Greene y Park, 2007; Miles y Stine-Morrow, 2004). Lo que plantean es que cambios en los mecanismos que subyacen a la autorregulación de la actuación lingüística serían fundamentales para explicar la mantención o el declive de las habilidades sintácticas en la vejez. Aunque la relación entre esos cambios y las modificaciones a nivel cerebral no está del todo clara, la hipótesis central es que los adultos mayores pueden mantener la eficacia de su actuación ajustando sus patrones de desempeño mediante estrategias que les permiten usar de manera más eficiente sus recursos de procesamiento disponibles. Sin embargo, esto podría ser más difícil para los adultos mayores con menos recursos de $\mathrm{MO}$ destinados al control ejecutivo de la actuación (StineMorrow et al., 2006b). Igualmente, discrepancias entre las capacidades efectivas y su apreciación por parte de los sujetos podrían conducir a confiar erradamente en las propias posibilidades de desempeño y así tomar decisiones no concordantes con las exigencias u objetivos de la tarea o bien a subestimar el propio potencial, lo que puede ocasionar cuadros de ansiedad frente a la tarea que conllevan a una pérdida de control sobre los propios procesos (Stine-Morrow et al., 2006a).

En general, las explicaciones basadas en mecanismos compensatorios han ido concitando gran atención en los estudios sobre lenguaje y envejecimiento. Esto ha llevado a considerar la importancia de determinados factores ambientales que favorecen la plasticidad funcional del cerebro en la vejez normal. Estos factores se vinculan fuertemente con el estilo y calidad de vida de los sujetos a lo largo de su existencia. Diferencias en este ámbito permitirían explicar la variabilidad encontrada en mediciones de diversas habilidades cognitivas y lingüísticas en personas de la tercera edad (Winfield y Grossman, 2006). Fundamental en este sentido es haber alcanzado un buen nivel educativo y ocupacional, así como la práctica continua de las habilidades intelectuales, pues todo ello permitiría contar con una reserva cognitiva que contribuiría a que el desempeño de las personas mayores no difiera significativamente del demostrado por los jóvenes (Rodríguez Álvarez y Sánchez Rodríguez, 2004). Una hipótesis concordante con esta idea, pero más específica, es la denominada hipótesis de la reserva (Payne, Gao, Noh, Anderson y Stine-Morrow, 2012; Stern, 2009), según la cual la mantención de hábitos de 
lectura a lo largo de la vida permite atenuar el declive general de la memoria (vg. Manly, Schupf, Tank y Stern, 2005) y ayuda a prevenir patologías cognitivas asociadas al envejecimiento (vg. Wilson, Bennett, Gilley, Beckett, Barnes y Evans, 2000).

Desde la perspectiva de la reserva, los posibles efectos del declive de la $\mathrm{MO}$ en el procesamiento sintáctico podrían moderarse o relativizarse en sujetos que se han mantenido activos intelectualmente a pesar de los años. Así, por ejemplo, los ya citados Payne et al. (2012), en un estudio sobre memoria de oraciones y análisis de tiempos de lectura, concluyen que un alto nivel de literacidad se relaciona con una mayor eficiencia de procesamiento y actúa como mecanismo compensatorio en adultos mayores con menor amplitud de MO. Por su parte, Goral et al. (2011) muestran que un alto nivel de educación es un predictor de buen desempeño en tareas de juicio frente a oraciones complejas de diverso tipo. En tanto, Caplan, Waters y Alpert (2003) encontraron que adultos mayores con un alto nivel educativo presentaban una velocidad de procesamiento y un patrón de activación del hemisferio cerebral izquierdo similar al de jóvenes con equivalentes años de estudio. En nuestro ámbito, Véliz, Riffo y Vásquez (2009) no encontraron diferencias asociadas a la edad ni a la memoria en una tarea de recuerdo y comprensión de oraciones realizada por senescentes y jóvenes con estudios superiores, pertenecientes al estrato social alto y medio alto. En un estudio anterior, también basado en el recuerdo de oraciones, Véliz (2004) halló que el desempeño de los adultos mayores del estrato alto era inferior al de los jóvenes del mismo estrato socioeconómico, pero superior al de los mayores y jóvenes del estrato social bajo.

\subsection{Complejidad sintáctica y MO}

Las estructuras sintácticas que han concitado mayormente el interés de la investigación sobre procesamiento de oraciones y envejecimiento son las cláusulas de relativo anidadas o incrustadas en posición central. Ello se debe a que el papel de la $\mathrm{MO}$ en el procesamiento sintáctico se ha relacionado estrechamente con el procesamiento de oraciones complejas, esto es, estructuras que involucran operaciones de cómputo e integración de información más demandantes (vg. Gibson, 1998); King y Just, 1991; Vos, Gunter, Kolk y Mulder, 2001). La explicación más común es que la interrupción de la cláusula principal exige más recursos de almacenamiento para mantener en la memoria la información lingüística mientras se completa el análisis (Miller y Chomsky, 1963; Frazier y Rayner, 1988), lo que no ocurre cuando la incrustación es a la izquierda.

Sin embargo, no es sólo el locus de incrustación lo que determina la dificultad de procesamiento, existen también diferencias asociadas a la función que cumple el pronombre relativo, si sujeto u objeto. Aunque estas diferencias se han relacio- 
nado con principios universales, algunos estudios muestran que también parecen depender de propiedades estructurales de las distintas lenguas. Así, mientras algunas investigaciones indican que en lenguas como el chino (Hsiao y Gibson, 2003, refutados posteriormente por Chier-Jer y Bever, 2006) y el vasco (Carreiras, Duñabeitia, Vergara, De la Cruz-Pavía y Laka, 2010) las relativas sujeto resultan más complejas de procesar que las relativas objeto, abundantes investigaciones empíricas referidas a distintas lenguas indoeuropeas muestran que cuando el pronombre cumple la función de objeto (El senador que acusó el diputado golpeó al fotógrafo), la carga de procesamiento es mayor que cuando el mismo cumple la función de sujeto (El senador que acusó al diputado golpeó al fotógrafo). Evidencias en este sentido se encuentran, entre otros, en Betancort, Carreiras y Sturt, 2009 y Del Río y López-Higes, 2006 (español); King y Just, 1991 y MacWinney y Pleh, 1988 (inglés); Holmes y O’ Regan, 1981 (francés); Frazier, 1987 y Mak, Vonk y Schriefers, 2002, 2006 (alemán). La comparación entre anidamiento de relativa sujeto y relativa objeto se ha convertido, en razón de lo anterior, en uno de los diseños experimentales clásicos en la investigación sobre $\mathrm{MO}$ y procesamiento sintáctico.

La asimetría entre relativas objeto y relativas sujeto se ha explicado desde diferentes enfoques. Desde uno que apela a preferencias de carácter más bien universal por la interpretación sujeto, una de las hipótesis más conocidas es la del cambio de perspectiva (MacWinney y Pleh, 1988). Según ésta, dado que el orden de la cláusula relativa objeto (OVS) no corresponde al canónico (SVO) y que el sujeto de la relativa objeto no es el mismo que el de la cláusula principal, se produce un cambio de perspectiva durante su análisis: desde el sujeto de la cláusula principal al sujeto de la relativa y desde éste nuevamente hacia el sujeto de la cláusula principal. Este cambio consumiría recursos, pues se requiere trasladar el foco de atención. Lo mismo ha sido explicado por autores como Caplan y Waters (1999) como un orden no canónico de los roles temáticos.

Otras hipótesis se enmarcan en un enfoque que enfatiza más en las características estructurales de las oraciones y relaciona de manera más explícita las operaciones implicadas en su análisis con el consumo de recursos de MO. Una de estas hipótesis es la de Clifton y Frazier (1989), quienes postulan que durante el procesamiento los hablantes aplican una estrategia destinada a economizar recursos cognitivos. Dicha estrategia consiste en llenar la huella dejada por una categoría vacía lo más rápidamente posible (active filler strategy), recurriendo para ello al análisis más fácilmente disponible. En las oraciones con anidamiento de relativa, esto consiste en asignar al pronombre relativo la función de sujeto. Si la función es la de objeto, la aplicación de esta estrategia conduce a un análisis incorrecto, por lo que se requiere posteriormente un reanálisis que aumenta la complejidad de todo el proceso.

En este mismo enfoque destaca también la llamada teoría de la localidad de las dependencias sintácticas, de Gibson y colaboradores (vg. Gibson, 1998; Gib- 
son, Desmet, Grodner, Watson y Ko, 2005). Esta teoría explica la complejidad de las relativas objeto como consecuencia de un mayor costo de las operaciones implicadas en su procesamiento: costos de memoria, relacionados con la mantención de dependencias temático-sintácticas incompletas, y costos derivados de la integración de los ítemes léxicos en los constituyentes y núcleos sintácticos de los cuales dependen, así como del número de referentes nuevos que son introducidos durante el análisis.

Con el fin de contribuir con datos provenientes del español a la discusión sobre los efectos del envejecimiento en el procesamiento de este tipo de oraciones, en este trabajo presentamos un estudio experimental en el que se compara el desempeño de jóvenes y adultos mayores. El experimento tuvo por objetivo poner a prueba la hipótesis del declive del procesamiento y comprensión de oraciones complejas en la vejez, a causa de la disminución de la capacidad de MO en los adultos mayores. Siguiendo esta hipótesis, los sujetos podrían alcanzar tiempos de lectura más altos que los jóvenes en las zonas críticas de las relativas objeto, esto es, en los segmentos oracionales cuyo procesamiento es más demandante. En relación con las respuestas, podrían presentar latencias más altas y porcentajes de respuestas correctas más bajos que los jóvenes en las mismas estructuras. Si, por el contrario, al comparar los grupos no se encuentran diferencias significativas asociadas a la edad y la MO, las evidencias podrían interpretarse como favorables a la hipótesis de Caplan y Waters (1999).

\section{MÉTODO}

\subsection{Participantes}

Participaron voluntariamente en el experimento 24 adultos mayores y 24 estudiantes universitarios de pregrado, con residencia en diferentes comunas de la Provincia de Concepción, Chile. Se consideró la edad como variable categórica: joven/adulto mayor, siguiendo la tradición de los estudios sobre envejecimiento y lenguaje que busca establecer los efectos que la pertenencia al grupo etario viejo tiene en la actuación lingüística. Se definió como criterio de corte para la selección de los participantes tener 65 años, pues la literatura indica que los cambios asociados al envejecimiento cognitivo, si bien son paulatinos, comienzan a notarse a contar de la sexta década de vida (vg. Junqué y Jodar, 1990, 1994).

Como una forma de evitar que el nivel socioeconómico afectara negativamente el desempeño en la tarea experimental, se decidió seleccionar sólo a sujetos del nivel socioeconómico medio o alto. Como medio de tamizaje se aplicó la Escala Graffar de clasificación socioeconómica. Además, se estableció como requisito contar con estudios superiores. 
La edad de los adultos mayores iba desde 65 a 81 años $(\overline{\mathrm{X}}=72, D T=4,0)$. Fueron reclutados en su mayor parte de cursos que ofrece la Universidad de Concepción al adulto mayor o de los registros de ex funcionarios administrativos o docentes de la misma universidad. A todos ellos se les realizó previamente una evaluación psicológica para descartar a quienes presentaran signos de déficit cognitivo o de alteración del ánimo, factores que también podían afectar el desempeño en la tarea experimental. Los instrumentos empleados con este propósito fueron el test de Bender-Bip y la Escala Abreviada de Depresión Geriátrica de Yesavage estandarizada para la población chilena (Quiroga y Fasce, 2002). Además, se les hizo llenar una ficha personal para verificar que mantenían hábitos de lectura.

Los estudiantes, alumnos de pregrado de dos universidades locales, pertenecían a distintas carreras en las que cursaban desde segundo año hacia arriba. Sus edades fluctuaban entre 19 y 30 años $(\overline{\mathrm{X}}=22, D T=3,2)$. En cuanto al nivel educativo, los adultos mayores tenían, en promedio, 16.2 años de educación formal, y los jóvenes, 15.8. Todos los participantes tenían vista normal o corregida.

La amplitud de $\mathrm{MO}$ se midió con la aplicación individual de una versión de la prueba de MO de Daneman y Carpenter (1980), especialmente adaptada al español de Chile por el equipo de investigación. De acuerdo a esta versión, los sujetos debían leer sucesivamente series de 2, 3, 4, 5 ó 6 oraciones, retener las últimas palabras de las oraciones de la serie y decidir, al mismo tiempo, si lo que decían las oraciones leídas era verdadero o falso. Además, se utilizó la prueba de memoria de corto plazo de dígitos del Test de Inteligencia para Adultos de Wechsler (WAIS).

\subsection{Estímulos experimentales}

Se construyeron 80 oraciones experimentales, la mitad en versión de relativa sujeto $(\mathrm{RS})$ y la otra mitad en versión de relativa objeto (RO). En la Tabla I se muestra un ejemplo de cada una de las dos condiciones sintácticas en estudio.

Tabla I. Ejemplo del tipo de oraciones experimentales.

\begin{tabular}{cc}
\hline Tipo de relativa & Oración \\
\hline RS & El senador [que acusó al diputado] golpeó al fotógrafo. \\
& V1 N2 V2 \\
\hline RO & El senador [que acusó el diputado] golpeó al fotógrafo. \\
V1 N2 V2
\end{tabular}

Las palabras en negritas corresponden a los segmentos cuyos tiempos de lectura fueron sometidos a análisis: el verbo y el sustantivo de la cláusula anidada (V1 y 
N2 respectivamente) y el verbo de la cláusula principal (V2). En V1 no se esperan diferencias asociadas al tipo de oración, ya que hasta ese punto las oraciones son iguales. En cambio, en los segmentos N2 y V2 podrían producirse diferencias entre los jóvenes y los adultos mayores que podrían relacionarse con la $\mathrm{MO}$ y la mayor complejidad de las relativas objeto en comparación con las relativas sujeto.

La longitud de la mayor parte de las oraciones experimentales fue de 9 palabras, pero aumentó a 10 u 11 por el uso de la preposición a cuando los sustantivos eran de género femenino, como en La niña que abrazó al payaso miró a la bailarina o El fotógrafo que sedujo a la modelo perdió a la novia.

Los estímulos se contrabalancearon de modo que cada sujeto leyera solo una versión de cada oración y recibiera igual número de los dos tipos de estructuras sintácticas, lo que dio como resultado 2 series de 40 oraciones. Además, se intercalaron 40 oraciones de relleno de longitud y estructura variadas. Los estímulos dentro de cada serie se ordenaron en forma aleatoria para ser presentados a los participantes. Todas las oraciones iban seguidas de una pregunta de comprensión que, en el caso de las oraciones experimentales, requería identificar al agente de la acción referida por el verbo principal. Los participantes fueron asignados al azar y en igual número a una de las dos series de estímulos (ver la lista completa de los estímulos en Anexos).

\subsection{Tarea experimental}

Las oraciones fueron presentadas en la pantalla de un computador para ser leídas mediante la técnica de ventana móvil. Para ello se utilizó una versión del programa computacional ERTS (Experimental Run-Time System, 3.32, BeriSoft Corporation). El sujeto se autoadministraba los estímulos palabra a palabra pulsando la barra espaciadora para avanzar. Al término de cada oración se mostraba en el centro de la pantalla una pregunta; debajo de ella, en el extremo inferior derecho del monitor, aparecía escrita la opción $S I ́$ y en el extremo izquierdo, la opción $N O$. El sujeto debía escoger una de las dos respuestas presionando una tecla verde $(S I ́)$ o roja $(N O)$. Registrada la respuesta, desaparecía la pregunta, se limpiaba la pantalla y se mostraba en el centro de ella una línea de asteriscos durante 1.800 ms, luego de lo cual se proseguía con el experimento. Para familiarizar a los participantes con la tarea, se les proporcionaron instrucciones verbales y 5 oraciones de ensayo antes de presentar las oraciones experimentales. Sólo después de estas oraciones se proporcionó feedback a través de un mensaje escrito en el centro de la pantalla: "BIEN" o "MAL", según la respuesta dada a la pregunta sobre la oración leída. Además, se programaron dos pausas de 15 segundos cada una durante la ejecución del experimento. Todas las sesiones experimentales se desarrollaron 
en el Laboratorio de Psicolingüística de la Universidad de Concepción. Una representación gráfica de los datos experimentales generados por el experimento se muestra en Anexos.

\subsection{Tratamiento de los datos}

La base de datos quedó constituida por las respuestas, tiempos de respuesta y tiempos de lectura registrados por los 24 jóvenes y 24 adultos mayores participantes, a un total de 1.920 oraciones. Para el tratamiento de los datos se tuvo en consideración que los tiempos de lectura y de respuesta no cumplían con la condición de tener una distribución normal; la variable correspondiente a las respuestas a las preguntas de comprensión es binaria (correcta o incorrecta) y, además, cada individuo debía responder 40 preguntas, es decir, las respuestas están agrupadas en grupos de 40, por lo que no cumplen con la condición de independencia. Dada estas condiciones, no son correctos el análisis de varianza ni la regresión lineal. Se optó entonces por los modelos lineales generalizados mixtos, que son formas avanzadas de regresión que permiten dar cuenta del comportamiento de datos con las características señaladas (Pinheiro y Bates, 2000; Richter, 2006; Baayen y Milin, 2010). Además, el interés del experimento es que la inferencia vaya dirigida a la población de sujetos con las mismas características, de la cual los individuos son considerados una muestra, un conjunto aleatorio de niveles del factor individuo. Por lo mismo, en los años recientes, estos modelos han ido ganando terreno en la psicolingüística experimental pues permiten analizar los efectos fijos y aleatorios considerando sujetos e ítemes de manera simultánea (como en efecto se hizo en el presente estudio), superando así al análisis por separado ( $F 1$ y F2) (Blozis y Traxler, 2007). Además, estas técnicas son capaces de modelar variables discretas y continuas simultáneamente, modelar diseños no balanceados o que no cumplen con las condiciones de normalidad o de homogeneidad de las varianzas, entre otros (Baayen, Davidson y Bates, 2008; Baayen y Milin, 2010).

Las mediciones tratadas como variables respuesta fueron los tiempos de lectura (tiempo total y de los puntos críticos en ms), tiempo de respuesta a la pregunta de comprensión (ms) y el acierto o fallo en la respuesta. En este último caso, la distribución de los datos fue binomial con función de enlace logit, mientras que para los tiempos de respuesta la distribución fue Gamma con función de enlace log. La variable predictor de interés fue la edad, conceptualizada como un factor de dos niveles: adulto joven/adulto mayor. Las demás variables fueron incorporadas como covariables predictoras: la amplitud de $\mathrm{MO}$ y la memoria de dígitos, ambas cuantitativas, y el tipo de oración (Tipo), conceptualizada como un factor de dos niveles: relativa objeto $(\mathrm{RO})$ y relativa sujeto $(\mathrm{RS})$. El modelo también incorporó 
como covariable la longitud media (LP) de cada uno de los segmentos críticos, la que fue medida en número de caracteres. Esto se hizo con el fin de controlar los posibles efectos intervinientes de las diferencias de longitud de las palabras sobre los tiempos de lectura. Para el caso de las variables tiempo de respuesta, acierto o error y tiempo de lectura total de las oraciones, la LP se calculó sumando el número de caracteres de todas las palabras críticas.

Para evaluar la significancia de los cinco potenciales predictores de las variables respuesta, primero ajustamos un modelo completo que incluía los efectos fijos y las interacciones de la variable edad con las covariables, además de la matriz de efectos aleatorios de sujetos e ítemes. Una vez obtenidos los efectos fijos que resultaron significativos, se construyó con ellos un modelo reducido para cada una de las variables respuesta. A partir de los modelos reducidos calculamos el tamaño del efecto del predictor significativo utilizando el inverso de la función de enlace y los coeficientes estimados de los predictores significativos. Cuando los dos predictores factoriales -edad (joven/adulto mayor) y tipo de oración (relativa sujeto/relativa objeto) - fueron significativos, calculamos el tamaño del efecto de cada uno de ellos para cada nivel del otro factor. En el caso de los predictores cuantitativos, el análisis se efectuó tomando como base sus valores promedio.

Todos los análisis fueron llevados a cabo en el ambiente y lenguaje de programación estadística $\mathrm{R}$ versión 2.5.12. Los gráficos fueron construidos utilizando el paquete de R lattice (Sarkar, 2008) y los modelo lineales generalizados mixtos fueron ajustados utilizando el paquete de R glmmADMB (Fournier, Skaug, Ancheta, Ianelli, Magnusson, Maunder, Nielsen y Sibert, 2012; Skaug, Fournier, Nielsen, Magnusson y Bolker, 2012).

\section{RESULTADOS}

Los resultados obtenidos con el primer modelamiento de los datos mostraron que no había interacciones entre la variable edad y las covariables sobre los tiempos de lectura, tiempos de respuesta y respuestas. Del mismo modo, los resultados también mostraron que no había efectos fijos de la $\mathrm{MO}$ sobre ninguna de las variables respuesta, por lo que las dos medidas que representaban esta covariable (amplitud de $\mathrm{MO}$ verbal y de dígitos) fueron desechadas del análisis. Pasamos entonces a probar los modelos reducidos para estimar el tamaño del efecto de cada uno de los predictores que sí resultaron significativos (grupo etario y covariables Tipo y LP).

La Tabla II muestra los valores de los coeficientes de los efectos fijos de los modelos seleccionados, el error estándar y la prueba de hipótesis de que estos efectos son significativos $(\mathrm{p}<0.05)$. 
Tabla II. Efectos fijos: edad, tipo de oración y LP.

\begin{tabular}{llcccc}
\hline Variable Respuesta & & \multicolumn{4}{c}{ Efectos Fijos } \\
\cline { 3 - 6 } & & Intercepto & Edad (AM*) & Tipo (RO) & LP \\
\hline Respuestas Correctas & Estimado & 2.986 & -0.661 & & \\
& Error Estándar & 0.212 & 0.279 & & \\
& valor p & $<0.01$ & 0.018 & & \\
\hline Tiempo de Respuesta & Estimado & 8.15 & 0.191 & 0.098 & \\
& Error Estándar & 0.059 & 0.082 & 0.024 & \\
& valor p & $<0.01$ & 0.020 & $<0.01$ & \\
\hline Tiempo Total & Estimado & 8.277 & 0.201 & 0.139 & 0.009 \\
& Error Estándar & 0.109 & 0.113 & 0.019 & 0.003 \\
& valor p & $<0.01$ & 0.077 & $<0.01$ & 0.002 \\
\hline Tiempo de Lectura V1 & Estimado & 5.611 & 0.58 & -0.06 & 0.022 \\
& Error Estándar & 0.117 & 0.146 & 0.028 & 0.009 \\
& valor p & $<0.01$ & $<0.01$ & 0.035 & 0.012 \\
\hline Tiempo de Lectura N22 & Estimado & 5.926 & & 0.062 & 0.194 \\
& Error Estándar & 0.124 & & 0.009 & 0.036 \\
& valor p & $<0.01$ & & $<0.01$ & $<0.01$ \\
\hline Tiempo de Lectura V2 & Estimado & 6.139 & & 0.058 & 0.292 \\
& Error Estándar & 0.119 & & 0.013 & 0.037 \\
& valor p & $<0.01$ & & $<0.01$ & $<0.01$ \\
\hline
\end{tabular}

* AM: adulto mayor.

El primer resultado que presenta la Tabla II corresponde al análisis de los efectos fijos del grupo etario, el tipo de oración y la longitud promedio de las palabras críticas sobre las respuestas dadas por los sujetos a las preguntas. Representa, por lo tanto, información sobre la comprensión de las oraciones a posteriori o fuera de curso. En este análisis sólo la variable edad mostró un efecto significativo ( $\mathrm{p}=$ 0.01) sobre la proporción de respuestas correctas: el grupo de adultos mayores obtuvo un coeficiente negativo del puntaje logit de -0.661 en comparación con los jóvenes.

En cuanto al tiempo o latencia de las respuestas, el grupo etario adulto mayor y las oraciones con $\mathrm{RO}$ presentan coeficientes positivos respecto del grupo de jóvenes y las RS respectivamente. Es decir, los adultos mayores tardaron más en responder que los jóvenes; a su vez, tanto jóvenes como adultos mayores presentan latencias de respuesta más largas en las relativas objeto. En ambos casos, el modelo arroja que se trata de efectos fijos altamente significativos. En relación con LP de las palabras críticas, los resultados indican que los tiempos de respuesta no tienen relación con esa covariable. 
En el tiempo de lectura total de las oraciones se observa un efecto sólo marginalmente significativo de la edad, como lo indica el valor p en la Tabla II (0.77). En cambio, y como era de esperarse, el tipo de oración resultó determinante en el comportamiento de esta variable respuesta $(\mathrm{p}=<0.01)$, resultado que confirma la mayor complejidad de las RO en comparación con las RS en español.

En relación con la covariable LP, los datos aportados por el modelo muestran que la longitud promedio de las palabras críticas también afecta el tiempo total. Es decir, se producen variaciones significativas $(\mathrm{p}=0.002)$ en los tiempos de lectura total cuando el número de caracteres de las palabras críticas aumenta o disminuye en relación al promedio (LP).

Sobre el tiempo de lectura de los puntos críticos, llama la atención que sólo en el primero, esto es, en el verbo de la cláusula de relativo (V1), se haya encontrado una diferencia significativa relacionada con la variable edad $(\mathrm{p}=<0.01)$. En cambio, en $\mathrm{N} 2$ y V2, en donde se produce una mayor carga de procesamiento, la edad no resultó ser un factor predictor. En cuanto al tipo de oración, si bien los datos muestran una diferencia estadísticamente significativa en el tiempo de lectura de $\mathrm{V} 1$ en las $\mathrm{RO}$, consideramos que se trata de un resultado que no guarda relación con el tipo de oración, ya que hasta el primer verbo las oraciones no se diferencian, tal como se puede comprobar si se revisa nuevamente los ejemplos de la Tabla I. En cambio, un dato que proporciona el modelo y que es independientemente del tipo de oración es que el efecto fijo de la covariable LP sobre el tiempo de lectura de V1 es significativo (0.012).

En los puntos críticos $\mathrm{N} 2$ y V2, los tiempos de lectura fueron concordantes con lo que las investigaciones señalan sobre la complejidad de las RO, pues la diferencia con las RS alcanzó un valor altamente significativo $(\mathrm{p}=0.001)$. La longitud promedio de las palabras afectó también el tiempo de lectura de estos dos puntos críticos. En el siguiente análisis se revisan los tamaños de los efectos significativos en sus unidades originales (proporción de respuestas correctas y tiempos de respuesta y lectura en milisegundos), lo que permite examinar mejor las diferencias relacionadas con la edad y el tipo de oración y evaluar qué pasa con ellas cuando se modelan considerando la covariable LP.

Tabla III. Tamaño de los efectos significativos.

\begin{tabular}{|llc|}
\hline Variable Respuesta & Condicionante & Efecto Edad \\
\hline Respuestas correctas & & -0.041 \\
\hline \multirow{2}{*}{ Tiempo de Respuesta } & RS & 729.800 \\
& RO & 805.302 \\
\hline \multirow{2}{*}{ Tiempo Total } & RS y LP & 1.103 .449 \\
& RO y LP & 1.267 .647 \\
\hline
\end{tabular}


Continuación Tabla III.

\begin{tabular}{|llc|}
\hline \multirow{2}{*}{ Tiempo de Lectura V1 } & RS y LP & 246.357 \\
& RO y LP & 232.016 \\
\hline \multirow{2}{*}{ Tiempo de Respuesta } & & Efecto Tipo \\
\hline \multirow{2}{*}{ Tiempo Total } & Joven & 358.211 \\
& Adulto Mayor & 433.713 \\
\hline \multirow{2}{*}{ Tiempo de Lectura V1 } & Joven y LP & 739.431 \\
& Adulto mayor y LP & 903.628 \\
\hline Tiempo de Lectura N2 & Joven y LP & -18.251 \\
\hline Tiempo de Lectura V2 & Adulto Mayor y LP & -32.592 \\
\hline
\end{tabular}

El tamaño del efecto de la edad sobre la comprensión de las oraciones indica que la tasa de éxito en las respuestas de los adultos mayores fue un $4 \%$ más baja que en los jóvenes. Para los tiempos de respuesta, las magnitudes de los coeficientes se traducen en que los adultos mayores tardaron más que los jóvenes en responder las preguntas referidas tanto a las RS (+730 ms) como a las RO (+805 ms). $\mathrm{Al}$ examinar estos tiempos en función de la covariable predictora Tipo, se observa que si bien ambos grupos etarios presentan tiempos de respuesta mayores cuando las oraciones son de tipo objeto, la diferencia entre RS y RO fue mayor para los adultos mayores que para los jóvenes (+434 ms versus $+358 \mathrm{~ms}$ ).

En relación al tiempo de lectura total de las oraciones, se observa que, manteniendo constante la covariable LP, la diferencia marginalmente significativa entre el grupo de edad adulto mayor y el grupo jóvenes se traduce en que los adultos mayores tardan 1.103 ms más que los jóvenes cuando la oración es de tipo sujeto y 1.268 ms más cuando es de tipo objeto. Ahora bien, si para el tiempo de lectura total se examina el tamaño del efecto de la complejidad sintáctica en cada uno de los niveles de la variable edad, tenemos que la diferencia entre relativas objeto y relativas sujeto en los jóvenes fue de $739.431 \mathrm{~ms}$ y en las personas mayores la misma diferencia fue 903.628.

Finalmente, para los tiempos de lectura de N2 y V2, la Tabla III confirma que a nivel de su LP estos segmentos demandan más tiempo de procesamiento en las $\mathrm{RO}$, confirmando la mayor complejidad de estas estructuras en comparación con las RS. 


\section{DISCUSIÓN FINAL}

El propósito de este estudio fue investigar si la edad y la amplitud de MO afectan el procesamiento de oraciones complejas en la vejez normal. El objetivo era poner a prueba la hipótesis que sostiene que la disminución de la capacidad de MO que experimentan las personas mayores afecta el procesamiento de oraciones de sintaxis compleja. Se emplearon oraciones con incrustación central de cláusula de relativo sujeto y objeto, lo que permitió estudiar los efectos de la complejidad en el procesamiento.

Para la selección de los participantes se tuvo en consideración los estudios que muestran o sugieren que el nivel educativo y sociocultural modulan los efectos del envejecimiento cognitivo (vg. los citados Payne et al., 2012; Véliz, 2004; Waters, Caplan, Alpert y Stanczak, 2003). Por lo tanto, se controlaron estas variables mediante la selección de participantes de nivel socioeconómico medio o alto, con estudios superiores (de nivel técnico, universitario o afines) y que se mantenían intelectualmente activos. De esta forma, se buscaba evitar que las posibles diferencias de desempeño entre jóvenes y adultos mayores respondieran a factores individuales distintos a la edad y la MO.

Se analizaron las mediciones efectuadas a la comprensión de las oraciones (respuestas a preguntas y sus correspondientes latencias) y al procesamiento inmediato (tiempos de lectura de puntos críticos y tiempo de lectura total) considerando la variable edad y las covariables memoria (span de memoria operativa verbal y de dígitos) y tipo de oración ( $\mathrm{RS}$ y $\mathrm{RO}$ ). Para controlar los posibles efectos de la longitud de las palabras críticas sobre las variables respuesta, en el modelamiento de los datos se introdujo la LP de las palabras críticas como covariable.

El examen de los datos obtenidos evidencia claramente que no hubo efectos de la $\mathrm{MO}$ en el desempeño de los sujetos en la tarea experimental. Es decir, la MO no resultó ser un predictor de los tiempos de procesamiento durante la lectura de las oraciones, como tampoco de la comprensión de éstas. Es probable que el nivel educativo de los participantes y la mantención de actividades intelectuales vinculadas con la lectura, se relacionen con este resultado.

En relación con la edad, el análisis muestra que los adultos mayores necesitaron más tiempo para responder las preguntas sobre las oraciones leídas y a su vez se equivocaron más al responder. Esto indica que el envejecimiento se relaciona con una menor eficiencia en el procesamiento a posteriori o fuera de curso. Cabe señalar que los efectos significativos de la edad fueron independientes del tipo de oración, pues no se encontraron interacciones entre ambas variables, como tampoco efectos fijos de la complejidad en las respuestas, aunque sí en las latencias. No obstante, al examinar el tamaño de los efectos fijos de la edad y la complejidad, los resultados son siempre más favorables para los jóvenes que para los adultos mayores. 
En contraste con los resultados obtenidos por los adultos mayores en la tarea offline, no se evidenciaron efectos significativos de la edad en los tiempos de lectura de las palabras donde se producen las operaciones más demandantes de recursos de memoria y cómputo sintáctico (N2 y V2). El análisis de estos datos hace pensar que una mayor complejidad sintáctica no representa necesariamente una mayor dificultad en el análisis sintáctico inmediato, a pesar de los cambios que provoca el envejecimiento a nivel cerebral. Por lo tanto, en términos generales, los resultados respaldan la hipótesis de la preservación en la tercera edad de los mecanismos básicos responsables del procesamiento sintáctico. En este sentido, el análisis es favorable a la posición de Caplan y Waters (1999): no se ha encontrado evidencias de declive en el procesamiento interpretativo como efecto de la disminución de la capacidad de MO.

No obstante lo anterior, un resultado que llama la atención es que en el verbo de la relativa los adultos mayores hayan registrado un tiempo de lectura más largo. Como se explicó, en V1 las oraciones aún no se diferencian, pues tanto en las RS como en las RO el orden oracional es el mismo. En V1 no es posible completar las operaciones de análisis conducentes a asignar los roles sintáctico-temáticos en la relativa, pues no se cuenta aún con la información necesaria para completar su procesamiento (recuérdese que la presentación de las oraciones fue palabra a palabra). Sólo la presencia o ausencia de la preposición a después de V1 indica si se trata de una RS o de una RO. ¿Qué podría explicar entonces la diferencia entre el tiempo de lectura de los jóvenes y los adultos mayores? Al respecto, parece necesario tener en cuenta que el procesamiento de las oraciones es en línea, por lo tanto opera de manera inmediata y progresiva. En este sentido, una hipótesis que podría contribuir a explicar nuestro hallazgo es la propuesta de Clifton y Frazier (1989), según la cual los sujetos realizan el análisis más fácilmente disponible y que conlleva el menor costo de procesamiento (active filler strategy). Por lo tanto, en V1 integrarían el pronombre relativo como sujeto de la relativa, operación que demanda mayor tiempo a los adultos mayores, cuya velocidad de procesamiento es afectada por el envejecimiento. Posteriormente, si en las oraciones con RO ese análisis no es reparado en N2, la representación global de la oración se ve afectada, lo que se refleja en un desempeño más descendido en la tarea de comprensión.

Sin embargo, el análisis de los resultados también indica que es necesario seguir profundizando en el estudio de los factores que inciden en el desempeño más bajo de los adultos mayores en la tarea off line. Al respecto, la revisión de la literatura deja en evidencia que el declive de la $\mathrm{MO}$ en la vejez se asocia con desempeños más bajos en diversas tareas de comprensión de oraciones (vg. Kemper et al., 2001; Kail et al., 2012; Goral et al., 2011). En este estudio, dicha variable no explica las diferencias significativas entre jóvenes y adultos mayores encontradas al comparar tiempos de respuesta y proporción de aciertos.

Es probable que el desempeño de los adultos mayores en la tarea de compren- 
sión así como la diferencia significativa con los jóvenes en el tiempo de lectura de V1 se relacione con su nivel educativo y la práctica continua de la lectura. Estos factores pueden haber favorecido su actuación, lo que podría contribuir a explicar el hecho de que en los puntos de mayor complejidad de procesamiento no mostraran diferencias significativas en comparación con los jóvenes, a pesar de su menor velocidad. Por su parte, el mayor tiempo que dedicaron al procesamiento de V1 así como su desempeño más bajo en las respuestas podrían reflejar cambios en la forma de afrontar la tarea experimental: más cautelosos al inicio probablemente como una manera de tratar de alcanzar un mejor desempeño en la tarea de comprensión, pues, al basarse en preguntas sobre las oraciones, los sujetos las podrían haber percibido como un examen a sus capacidades. Esto mismo quizás podría haber contribuido a obtener finalmente una tasa de error mayor que la de los jóvenes. Desde esta perspectiva, los planteamientos de Stine Morrow y colaboradores sobre cambios en los mecanismos que subyacen a la autorregulación de la actuación lingüística en las personas mayores representan una alternativa promisoria para el desarrollo de nuevas investigaciones sobre la actuación lingüística en la tercera edad.

Finalmente, el experimento realizado aporta nueva información que confirma la asimetría entre el procesamiento de relativas sujeto y objeto. En efecto, la covariable que ejerció un efecto más robusto en el comportamiento de los datos fue el tipo de oración. Salvo en las respuestas, en todas las mediciones las oraciones con relativa objeto fueron más complejas que las relativas sujeto, resultados que concuerdan con los estudios llevados a cabo por diversos autores.

\section{REFERENCIAS}

Baayen, R. H. y Milin, P. (2010). Analyzing reaction times. International Journal of Psychological Research, 3(2), 12-28.

Baayen, R. H., Davidson, D. J. y Bates, D. M. (2008). Mixed-effects modeling with crossed random effects for subjects and items. Journal of Memory and Language, 59, 390-412.

Baddeley, A. D. (1986). Working memory. Oxford: Oxford University Press.

Baddeley, A. D. y Hitch, G. 1974. Working memory. En G. H. Bower (ed.), The psychology of learning and motivation. Vol. 8: 47-89. Nueva York: Academic Press.

Baltes, P. y Baltes, M. M. (eds.). (1990). Successful aging: Perspectives from the behavioral sciences. New York: Cambridge University Press.

Betancort, M., Carreiras, M. y Sturt, P. (2009). The processing of subject and object relative clause in Spanish: An eye-tracking study. Quarterly Journal of Experimental Psychology, 62, 1915-1929. 
Blozis, S.A. y Traxler, M. J. (2007). Analyzing individual differences in sentence processing performance using multilevel models. Behavior Research Methods, 39(1), 31-38.

Buckner, R. (2004). Memory and executive function in aging and AD: multiple factors that compensate. Neuron, 44(1), 195-208.

Burke, D. M. y Shafto, M. A. (2008). Language and aging. En F. I. M. Craik y T. A. Salthouse (eds.), The handbook of aging and cognition (pp. 373-443). New York: Psychology Press.

Cabeza, R. (2002). Hemispheric asymmetry reduction in older adults: The Harold model. Psychology and Aging, 17, 85-100.

Cabeza, R. Anderson, N. D., Locantore, J. K. y McIntosh, A. R. (2002). Aging gracefully: Compensatory brain activity in high-performing older adults. NeuroImage, 17, 1394-1402.

Cabeza, R., Grady, C. L., Nyberg, L., McIntosh, A. R., Tulving, E. y Kapur, S. (1997). Age-related differences in effective neural connectivity during encoding and retrieval: A possitron emission tomography study. Journal of Neurosciencie, 17, 391-400.

Caplan, D. y Waters, G. (1999). Verbal working memory and sentence comprehension. Behavioral and Brain Sciences, 22, 77-126.

Caplan, D., Waters, G. y Alpert, N. (2003). Effects of age and speed of processing on rCBF correlates of syntactic processing in sentence comprehension. Human Brain Mapping, 19, 112-131.

Caplan, D., Dede, G., Waters, G., Michaud, J. y Tripodis, Y. (2011). Effects of age speed of processing, and working memory on comprehension of sentences with relative clauses. Psychology and Aging, 26(2), 439-50.

Carpenter, P.A., Miyake, A. y Just, M.A. (1994). Working memory constraints in comprehension. Evidence from individual differences, aphasia, and aging. En M. A. Gernsbacher (ed.), Handbook of Psycholingüistics (pp. 1075-1122). San Diego, California: Academic Press, Inc.

Carreiras, M., Duñabeitia, J. A., Vergara, M., De la Cruz-Pavía, I. y Laka, I. (2010). Subject relative clauses are not universally easier to process: Evidence from Basque. Cognition, 115, 79-92.

Cerella, J. (1985). Information processing rates in the elderly. Psychologycal Bulletin, 98, 67-83.

Clifton Jr, C. y Frazier, L. (1989). Comprehending sentences with long-distance dependencies. En G. N. Carlson y M. K. Tanenhaus (eds.), Linguistic structure in language processing (pp. 273-317). Dordrecht: Kluwer.

Clifton, Ch., Traxler, M., Mohamed, M., Williams, R., Morris, R. y Rayner, K. (2003). The use of thematic role information in parsing: Syntactic processing autonomy revisited. Journal of Memory and Language, 49, 317-344.

Craik, F. I. M., Anderson, N. D., Kerr, S. A. y Li, K. Z. H. (1995). Memory 
changes in normal aging. En A. D. Baddeley, B. A. Willson y F. N. Watts (eds.), Handbook of memory disorders (pp. 211-241). Nueva York: John Wiley and Sons.

Cheung, H. y Kemper, S. (1992). Competing complexity metrics and adults production of complex sentences. Applied Psycholinguistics, 13, 53-76.

Chier-Jer, C.L. y Bever, T. (2006). Subject preference in the processing of relative clauses in Chinese. En D. Baumer, D. Montero and M. Scanlon (eds.), Proceedings of the 25th West Coast Conference on Formal Linguistics (pp. 254-260). Somerville, MA: Cascadilla Proceedings Project.

Daneman, M. y Carpenter, P. (1980). Individual diferences in working memory and reading. Journal of Verbal Learning and Verbal Behavior, 19: 450-466.

Dede, G., Caplan, D., Kemtes, K. y Waters, G. (2004). The Relationship Between Age, Verbal Working Memory, and Language comprehension. Psychology and Aging, 19(4), 601-616.

Del Río, D. y López-Higes, R. (2006). Efectos de la memoria operativa y de una carga de procesamiento en la comprensión de oraciones. Psicológica, 27, 79-95.

Escobar, A. (2001). Envejecimiento cerebral normal. Revista Mexicana de Neurociencia, 2(4), 197-202.

Fournier, D. A., Skaug, H. J., Ancheta, J., Ianelli, J., Magnusson, A., Maunder, M., Nielsen, A. y Sibert, J. (2012). AD ModelBuilder: using automatic differentiation for statistical inference of highly parameterized complex non linear models. Optimization Methods and Software, 27, 233-249.

Frazier, L. (1987). Syntactic processing: Evidence from Dutch. Natural Language y Linguistic Theory, 5(4), 519-559.

Frazier, L. y Rayner, K. (1988). Parameterizing the language processing system: le$\mathrm{ft}$-versus right-branching within and across languages. En J. A. Hawkens (ed.), Explaining language universals (pp. 247-279). Oxford: Blackwell.

Gibson, E. (1998). Linguistic complexity: locality of syntactic dependencies. Cognition, 68, 1-76.

Gibson, E., Desmet, T., Grodner, D., Watson, D. y Ko, K. (2005). Reading relative clauses in English. Cognitive Linguistics, 16(2), 313-353.

Gil, G. P. (2000). Bases fisiológicas del envejecimiento cerebral. Rev Mult Gerontol, 10(2), 67-91.

Goral, M., Clark-Cotton, M., Spiro, A., Obler, L. K, Verkuilen, J. y Albert, M. L. (2011). The contribution of set switching and working memory to sentence processing in older adults. Experimental Aging Research, 37(5), 516-538.

Grossman, M., A. Cooke, Ch. DeVita, D. Alsop, J. Detre, W. Chen y J. Gee. (2002). Age-related changes in working memory during sentence comprehension: An fMRI study. NeuroImage, 15, 302-317.

Hsiao, F. y Gibson, E. (2003). Processing relative clauses in Chinese. Cognition, 90, 3-27. 
Holmes, V. M. y O’Regan, J. K. (1981). Eye fixation patterns during the reading of relative clause sentences. Journal of verbal Learning and Verbal Behavior, 20, 417-430.

Junqué, C. y Jodar, M. (1990). Velocidad de procesamiento cognitivo en el envejecimiento. Anales de psicología, 6(2), 199-207.

Junqué, C. y Jurado, M. (1994). Envejecimiento y demencias. Barcelona: Martínez Roca.

Jurado, M. B., Matute, E. y Rosselli, M. (2008). Las funciones ejecutivas a través de la vida. Revista Neuropsicología, Neuropsiquiatría y Neurociencias, 8(1), 2346.

Just, M. A. y Carpenter, P. A. (1992). A capacity theory of comprehension: individual differences in working memory. Psychological Review, 99, 122-149.

Kail, M., Lemaire, P. y Lecacheur, M. (2012). Online grammaticality judgments in French young and older adults. Experimental Aging Research: An International Journal Devoted to the Scientific Study of the Aging Process, 38(2), 186-207.

Kemmer, L., Coulson, S., De Ochoa, E. y Kutas, M. (2004). Syntactic processing with aging: An event-related potencial study. Psychophysiology, 41, 372-384.

Kemper, S. (1987). Life-span changes in syntactic complexity. Journal of Geronto$\log y, 42,323-328$.

Kemper, S. (1992). Language and aging. En F. I. M. Craik y T. Salthouse (eds.), The handbook of aging and cognition (pp. 213-270). Hillsdale N. J.: Erlbaum.

Kemper, S. y Kemptes, K. (1999). Limitations on syntactic processing. En S. Kemper y R. Kliegl (eds.), Constraints on Language. Aging, grammar and memory (pp. 79-106). Norwell: Kluwer.

Kemper, S., Crow, A. y Kemptes, K. (2004). Eye-Fixations patterns of high-and low- span young and older adults: Down the garden path back again. Psychology and Aging, 1(19), 157-170.

Kemper, S., Greiner, L. H., Marquis, J. G., Prenovost, K. y Mitzner, T. (2001). Language decline across the life span: Findings from the nun study. Psychology and Aging, 16(2), 227-239.

Kemptes, K. y Kemper, S. (1997). Younger and older adults' on-line processing of sintactically ambiguous sentences. Psychology and Aging, 12, 362-371.

King, J. y Just, A. (1991). Individual differences in syntactic processing: the role of working memory. Journal of Memory and Language, 30, 580-602.

Kynette, D. y Kemper, S. (1986). Aging and the loss of grammatical forms: A cross-sectional study of language performance. Language and Communication, 6, 43-49.

MacWinney, B. y Pleh, C. (1988). The processing of restrictive relative clauses in Hungarian. Cognition, 29, 95-141.

Mak, W.M., Vonk, W. y Schriefers, H. (2002). The influence of Animacy on relative clause processing. Journal of Memory and Language, 47, 50-68. 
Mak, W. M., Vonk, W. y Schriefers, H. (2006). Animacy in processing relative clauses: The hikers that rocks crush. Journal of Memory and Language, 54(4), 466-490.

Manly, J. J., Schupf, N., Tank, M. X. y Stern, Y. (2005). Cognitive decline and literacy among ethnically diverse elders. Journal of Geriatric Psychiatry and Neurology, 18, 213-217.

Miles, J. R. y Stine-Morrow, E. A .L. (2004). Adult age differences in self-regulated learning in reading sentences. Psychology and Aging, 19, 626-636.

Miller, G. y Chomsky, N. (1963). Finitary models of language users. En R. D. Luce, R. Bush y E. Galanter (eds.), Handbook of Mathematical Psychology (pp. 269-321). Volume 2. New York, NY: Wiley.

Obler, L. K., Fein, D., Nicholas, M. y Albert, M. L. (1991). Auditory comprehension and aging: Decline in syntactic processing. Applied Psycholinguistics, $12,433-452$.

Park, D. C. (2000). The basic mechanisms accounting for age-related decline in cognitive function. En D. C Park y N. Schwarz (eds.), Cognitive aging: A Primer (pp. 3-22). Philadelphia: Taylor and Francis Group.

Park, D. y Payer, D. (2001). Working memory across the adult lifespan. En E. Bialystok y F. I. M. Craik (eds.), Lifespan cognition: Mechanisms of change (pp. 128-142). New York: Oxford University Press.

Payer, D., Marshuetz, CH., Sutton, B., Hebrank, A., Welsh R. C. y Park, D. (2006). Decreased neural specialization in old adults on a working memory task. NeuroReport, 5, 487-491.

Payne, B., Gao, X., Noh, S. R., Anderson, C. J. y Stine-Morrow, E. A. (2012). The effects of print exposure on sentence processing and memory in older adults: Evidence for efficiency and reserve. Aging, Neuropsychology, and Cognition. A Journal on Normal and Dysfunctional Development, 19(1-2): 122-149.

Pinheiro, J. C. y Bates, D. M. 2000. Mixed-effects models in S and S-PLUS. Statistics and Computing Series. New York: Springer-Verlag.

Quiroga, P. y Fasce, M. (2002). Evaluación psiquiátrica del adulto mayor. En P. Quiroga y G. Rohde (eds.), Psicogeriatría. Bases conceptuales. Clínica y terapéutica integral (pp. 67-111). Chile, Ediciones de la Sociedad de Neurología, Psiquiatría y Neurología.

Richter, T. (2006). What is wrong with ANOVA and multiple regression? Analyzing sentence reading times with hierarchical linear models. Discourse Processes, 41(3), 221-250.

Rodríguez Álvarez, F. y Sánchez Rodríguez, J. (2004). Reserva cognitiva y demencia. Anales de psicología, 20(2): 175-186.

Salthouse, T. (1985). A theory of cognitive aging. Amsterdam: North-Holland.

Salthouse, T. (1996). The processing-speed theory of adult age differences in cognition. Psychological Review, 103, 403-428. 
Sarkar, D. (2008). Lattice: Multivariate data visualization with R. New York: Springer - Verlag.

Skaug, H., Fournier, D., Nielsen, A., Magnusson, A. y Bolker, B. (2012). Generalized linear mixed models using $A D$ model builder. $\mathrm{R}$ package version 0.7.3.

Stern, Y. 2009. Cognitive reserve. Neuropsychologia, 47, 2015-2028.

Stine-Morrow, E. A. L. (2007). The Dumbledore hypothesis of cognitive aging. Current Directions in Psychological Science, 16(6), 295-299.

Stine-Morrow, E. A. L., Miller, L. y Hertzog, C. (2006a). Aging and self-regulated language processing. Psychological Bulletin, 132(4), 582-606.

Stine-Morrow E. A. L., Ryan, S. y Leonard, J. S. (2000). Age differences in on-line syntactic processing. Experimental Aging Research, 26, 315-322.

Stine-Morrow, E. A. L., Shake, M. C., Miles, J. R. y Noh, S. R. (2006b). Adult age differences in the effects of goals on self-regulated sentence processing. Psychology and Aging, 21(4), 790-803.

Stine-Morrow, E. A. L., Parisi, J. M., Morrow, D. G., Greene J. y Park, D. (2007). An engagement model of cognitive optimization through adulthood. Journals of Gerontology: Series B, 62, 62-69.

Tyler, L. K., Shafto, M. A., Randall, B., Wright, P., Marslen-Wilson, W. y Stamatakis, E. (2010). Preserving syntactic processing across the adult life span: The modulation of the frontotemporal language system in the context of agerelated atrophy. Cerebral Cortex, 20, 352-364.

Véliz, M. (2004). Procesamiento de estructuras sintácticas complejas en adultos mayores y adultos jóvenes. Estudios Filológicos, 39, 66-81.

Véliz, M, Riffo, B. y Vásquez, A. (2009). Recuerdo inmediato de oraciones de sintaxis compleja en adultos jóvenes y mayores. Estudios Filológicos, 44, 243-258.

Véliz, M., Riffo, B. y Arancibia, B. (2010). Envejecimiento cognitivo y procesamiento del lenguaje: Cuestiones relevantes. RLA 48(1), 75-105.

Vos, S. H., Gunter, T. C., Kolk, H. H. J. y Mulder, G. (2001). Working memory constraints on syntactic processing: An electrophysiological investigation. Psychophysiology, 38, 41-63.

Waters, G. y Caplan, D. (1999). Verbal working memory capacity and on-line sentence processing efficiency in the elderly. En S. Kemper y R. Kliegl (eds.), Constraints on Language. Aging, grammar and memory (pp. 107-136). Boston: Kluwer Academic Publishers.

Waters, G. y Caplan, D. (2001). Age, working memory and on-line syntactic processing in sentence comprehension. Psychology and Aging, 16, 128-144.

Waters, G., Caplan, D., Alpert, N. y Stanczak, L. (2003). Individual differences in rCBF correlates of syntactic processing in sentence comprehension: effects of working memory and speed of processing. NeuroImage, 19, 101-112.

Wilson, R. S., Bennett, D. A., Gilley, D. W., Beckett, L. A., Barnes, L. L. y Evans, D. A. (2000). Premorbid reading activity and patterns of cognitive decline in 
Alzheimer Disease. Archives of Neurology, 57, 1718-1723.

Winfield, A. y Grossman, M. (2006). Language and the aging brain: patterns of neural compensation revealed by functional brain imaging. J. Neurophysiol, 96(6), 2830-2839.

ANEXOS
Oraciones experimentales
El carabinero que detuvo al / el automovilista examinó al herido.
El senador que acusó al / el diputado golpeó al fotógrafo.
La niña que abrazó al /el payaso miró a la bailarina.
La profesora que acompañó al / el alumno esperó al director.
El ejecutivo que besó a la / la secretaria perdió al cliente.
El corresponsal que interrogó al / el soldado descubrió al espía.
El cura que visitó al / el obispo bendijo al niño.
El bombero que saludó al / el ciclista salvó al niño.
La madre que llamó a la / la niñera lavó al bebé.
El boxeador que recomendó al / el entrenador golpeó al amigo.
El investigador que citó a la / la mujer siguió al sospechoso.
El obrero que agredió al / el capataz dejó a la esposa.
El corredor que derrotó al / el australiano superó al campeón.
El cliente que esperó a la / la vendedora olvidó a la niña.
El chofer que vio al / el pasajero identificó al traficante.
El médico que esperó al / el paciente encontró a la enfermera.
El atleta que saludó al / el Presidente venció al oponente.
El investigador que citó al / el gerente descubrió al ladrón.
El ministro que denunció al / el alcalde recibió al periodista.
La señora que recomendó al / el jardinero cuidó al pequeño.
El ladrón que sorprendió al / el guardia detuvo a la cajera.
La señora que cuidó al / el anciano atendió al herido.
La dama que aplaudió al / el poeta visitó al escritor.
El reo que hirió al / el gendarme recibió al abogado.
El fotógrafo que sedujo a la / la modelo perdió a la novia.
El caballero que defendió al / el rey rescató a la doncella.
El malhechor que hirió al / el policía entregó al cómplice.
El concursante que abrazó al / el animador miró a la rubia.
El mago que engañó a la / la bruja hechizó al príncipe.
El terrorista que amenazó al / el piloto olvidó a la azafata.
El peatón que culpó al / el conductor presentó al testigo.


El músico que inspiró a la / la pianista escuchó al tenor.

El ajedrecista que defendió al / el maestro estudió al adversario.

El alumno que ofendió a la / la directora ignoró al profesor.

El escritor que felicitó al / el pintor observó a la modelo.

El hombre que vio al / el guardia asaltó a la ejecutiva.

La periodista que llamó al / el director ocultó al informante.

El garzón que atendió a la / la joven recordó al actor.

El abogado que irritó al / el juez objetó al testigo.

El inglés que atacó al / el pirata salvó a la princesa.
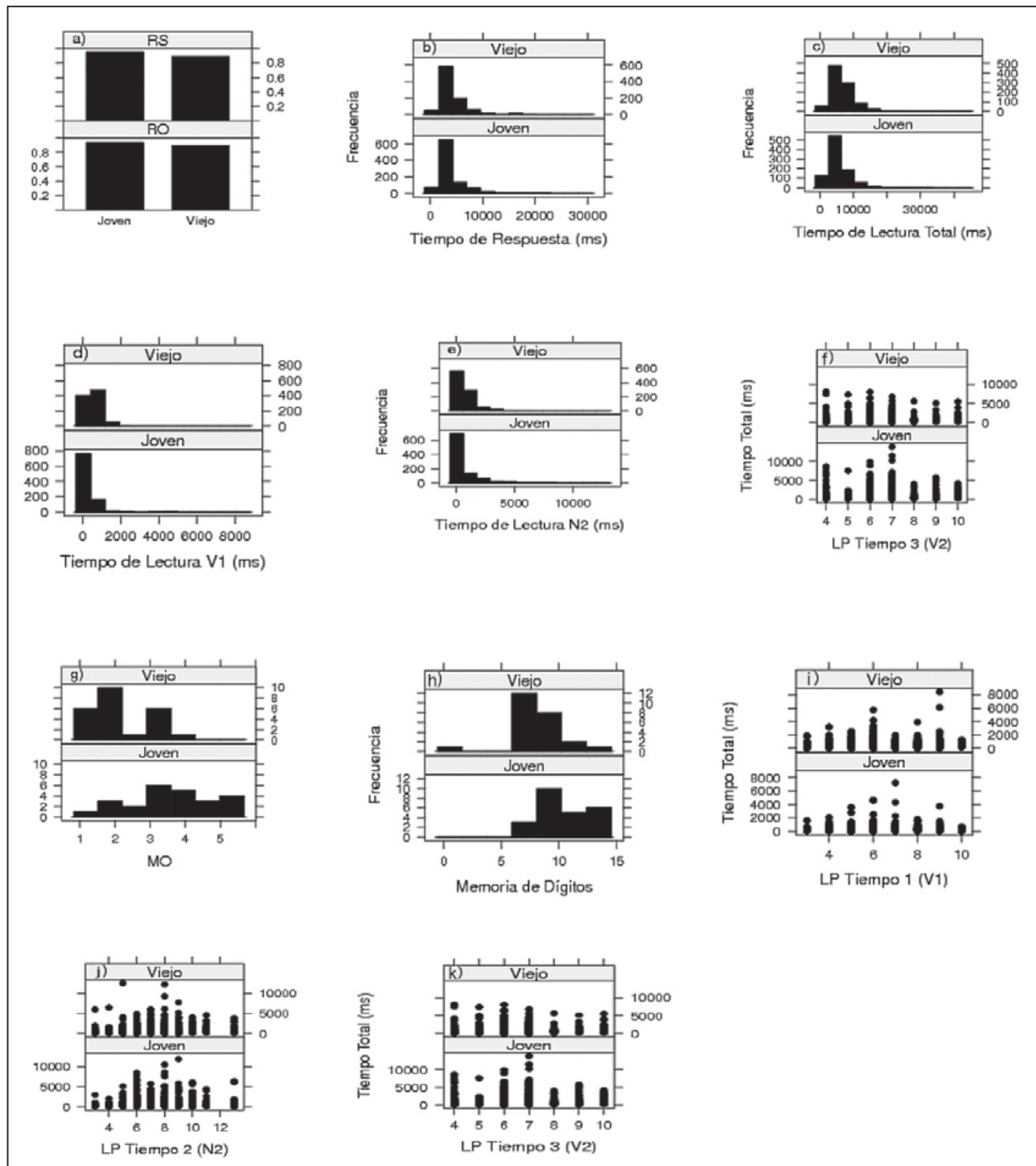

Figura 1. Descripción de los datos experimentales. 